\title{
LOCALIZED SPIN RELAXATION DUE TO COUPLING TO EFFECTIVE MASS STATES*
}

\author{
Z. WILAMOWSKI, J.E. DMochowsKI \\ Institute of Physics, Polish Academy of Sciences \\ Al. Lotników 32/46, 02-668 Warsaw, Poland \\ AND W. JANTSCH \\ Johannes Kepler Universität, 4040 Linz, Austria
}

The EPR of residual $\mathrm{Mn}$ in $\mathrm{CdF}_{2}$ doped with $\mathrm{Y}, \mathrm{In}$ and $\mathrm{Ga}$ is investigated. Although these donors are barely seen in EPR, they manifest themselves by a new effect: a drastic resonant reduction in the longitudinal relaxation rate of $\mathrm{Mn}$ which occurs only if the Zeeman splitting of the two subsystems coincide. In this situation, the saturation of those of the six $\mathrm{Mn}$ hyperfine lines is weakened which coincide with the shallow donor resonance.

PACS numbers: 75.10.Jm, 75.50.-y

$\mathrm{CdF}_{2}$ has an energy gap of more than $8 \mathrm{eV}$ and still it can be $n$-type doped semiconductor. Some of the donors investigated so far, In and Ga, exhibit also a deep state in addition to the shallow one which is the ground state for $Y[1,2]$. For In and $\mathrm{Ga}$, the shallow state can be populated by optical excitation and it remains in this metastable state below a donor-dependent critical temperature, just like in the case of the DX centres in AlGaAs. For Ga, this temperature is close to room temperature which makes this material an interesting candidate for memory devices making use of a variety of persistent optical and electronic effects [2-4]. The character of the metastable shallow donor state and the large lattice relaxation as the origin of the deep state are well established [5]. The nature of the deep state, however, is still controversial. In particular, there is still a discussion on whether the deep state is due to a negative $U$ centre with two electrons, like in the case of the DX centres in III-V compounds [6], or whether it is a one-electron positive $U$ center as described by the Toyozawa model [5]. In the latter case, the deep state should be observable by electron paramagnetic resonance.

In this paper, we present results of an investigation of $\mathrm{Y}$-, In- and Ga-doped $\mathrm{CdF}_{2}$ crystals by EPR. We observe the dominant spectra of $\mathrm{Mn}$, but no sign of a deep donor state. After photoexcitation to the metastable donor state, the saturation behaviour of some part of the $\mathrm{Mn}$ spectrum changes. We explain this effect in terms of an additional spin relaxation channel due to exchange coupling between the $\mathrm{Mn}$ to the delocalized shallow donor state.

*Work supported by the Committee for Scientific Research (Poland) grant No. 2P03B19108 and by the Fonds zur Förderung der Wissenschaftlichen Forschung (Austria). 
We investigate Bridgman grown samples of $\mathrm{CdF}_{2}$ doped with $\mathrm{Y}, \mathrm{Ga}$ or In during growth. The unintentionally incorporated $\mathrm{Mn}$ varies in concentration from sample to sample in the range of $1.5 \times 10^{18} \mathrm{~cm}^{-3}$. As grown samples are semi-insulating. To activate the donors, the samples are "converted" as described in the literature [1].

In Y-doped samples, which do not show metastability, a single line is seen $\left(g_{\mathrm{Y}}=1.956\right)$ in addition to the well resolved hyperfine (hf), superhyperfine (shf) and fine structure (fs) lines of $\mathrm{Mn}$. We attribute this line to the shallow donor state since this line exhibits motional narrowing above $140 \mathrm{~K}$ which can be explained by hopping of effective mass electrons from donor site to donor site.

Ga- or In-doped samples show semiconducting properties with giant persistent photoconductivity below $200 \mathrm{~K}$. In EPR we see again the well resolved $\mathrm{Mn}$ spectrum but only a very weak line due to the effective mass (shallow bound) electrons with $g$-factors of $g_{\mathrm{Ga}}=1.952 \pm 0.005$ and $g_{\mathrm{In}}=1.967 \pm 0.005$, respectively. The barrier between the deep and the shallow state is smaller for In and persistent effects are observed only below $70 \mathrm{~K}$. The shallow donor EPR is thus weak above $70 \mathrm{~K}$ because of the small population of the shallow states and the big line width.

The main result of this paper concerns the saturation behaviour of the Mn EPR spectra at high microwave power, $P$, which reflects the behaviour of the spin-lattice relaxation time. The saturation behaviour is very different for different samples and for a given sample it depends on temperature, illumination and for conducting samples it depends also on the part of the spectrum looked at: it differs for the different EPR lines of Mn.

The inset of Fig. 1 shows the dependence of the shf line amplitude of Mn on the microwave powerof a $\mathrm{Ga}$ doped sample at $300 \mathrm{~K}$ (triangles) and at $4 \mathrm{~K}$

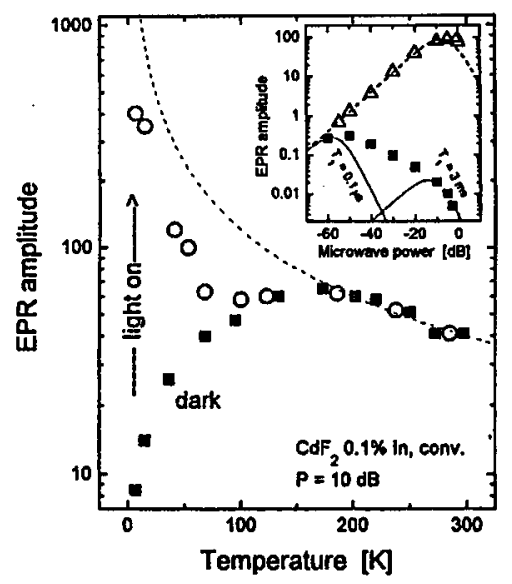

Fig. 1. Amplitude of Mn EPR lines in dark (solid squares) and after illumination (open circle) as a function of temperature for $\mathrm{CdF}_{2}: \mathrm{In}$. The dashed line corresponds to the unsaturated amplitude. The inset shows the dependence of the amplitudes of shf lines of $\mathrm{Mn}$ as a function of microwave power for a Ga doped sample: Triangles: $300 \mathrm{~K}$, squares: $5 \mathrm{~K}$. 


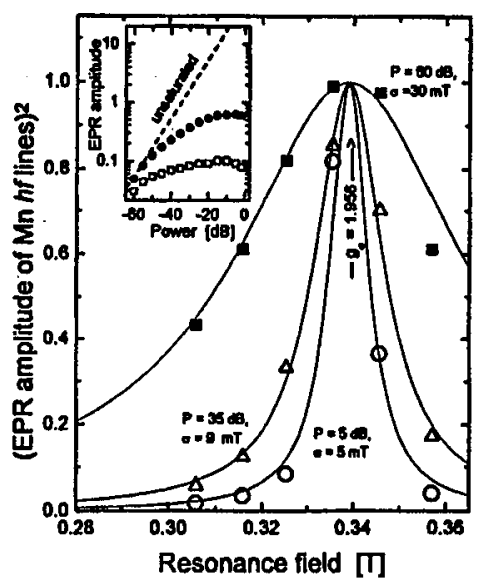

Fig. 2. Amplitudes of six hf EPR lines of $\mathrm{Mn}$ in $\mathrm{CdF}_{2}: \mathrm{Ga}$ plotted as a function of resonance field. Different symbols correspond to different microwave power, 60,35 and $5 \mathrm{~dB}$. The solid curves correspond to Lorentz line shapes, of different line width $(\sigma=30$, 9 , and $5 \mathrm{mT}$ ), all are centred at the shallow donor $g$-factor, $g_{\mathrm{s}}=1.956$. The inset shows a power dependence of first (open squares) and fourth hf lines.

(squares). For comparison saturation curves are calculated according to the Bloch equations using a single longitudinal relaxation time $T_{1}$ (left curve $3 \mathrm{~ms}$ and right curve $0.1 \mu \mathrm{s}$ ). The appearance of wide plateaus indicates inhomogeneous broadening and nonuniform distribution of longitudinal relaxation rates [7]. Figure 1 shows the temperature dependence of the amplitude of the hf line close to $g=2$ before (squares) and after (circles) illumination for a constant microwa ve power of $20 \mathrm{~mW}$. Below the metastability point of the In donor the signal increases persistently by up to two orders of magnitude showing clearly that we are dealing with an effect of the shallow donors on the Mn EPR.

The nature of this interaction becomes more clear if we compare the influence on the different parts of the spectrum as illustrated in Fig. 2, where the $6 \mathrm{Mn}$ hf line amplitudes (normalized) are shown after photoexcitation (before the 6 lines are equal in amplitude). The power dependence, given in the inset of Fig. 2, demonstrates different saturation behaviour. With increasing microwave power the outer hf lines in Fig. 2 saturate much faster than the line closest to the shallow donor $g$-factor. Obviously the longitudinal relaxation rate is strongly enhanced due to the coincidence of the Zeeman energy of the shallow donor and this particular $\mathrm{Mn}$ hf line. Connecting the data points we can fit a Lorentz line shape which is exactly centred at the shallow donor resonance.

The experimental results presented here show that there is a cross relaxation mechanism between the Mn $3 d^{5}$ states and the shallow donor state. If the shallow donor states are populated, those parts of the Mn EPR signal increase which have the same Zeeman splitting. From the dependence of this effect on microwave power we see that it is caused by a shortening of the longitudinal relaxation time of the $\mathrm{Mn}$ - obviously a new resonant relaxation path is opened by the overlap of the 
highly localized Mn state with long intrinsic $T_{1}$ and the delocalized shallow donor state with much shorter $T_{1}$. This effect allows us to detect the shallow donor EPR with much better sensitivity than by direct observation.

The present results support also the negative $U$ model for the Ga- and In donors in an indirect way. Even for the highest donor concentrations we observe neither the cross relaxation effect nor a direct EPR feature which could be ascribed to a paramagnetic deep state. The lack of the cross relaxation effect could be explained within a positive $U$ model by assuming a long $T_{1}$, but in that case we should be able to see the EPR directly. Either effect should be seen and since this is not the case the present experiments strongly support the diamagnetic ground state, i.e., the negative $U$ case also for the metastable donors in $\mathrm{CdF}_{2}$ as postulated already by Ryskin et al. [3], based on the observation of an extra contribution to the magnetic susceptibility after photoexcitation.

\section{References}

[1] U. Piekara, J.M. Langer, B. Krukowska-Fulde, Solid State Commun. 23, 583 (1977).

[2] J.E. Dmochowski, W. Jantsch, D. Dobosz, J.M. Langer, Acta Phys. Pol. A 73, 247 (1988).

[3] A.I. Ryskin, A.S. Shcheulin, B. Koziarska, J.M. Langer, A. Suchocki, I.I. Buczinskaya, P.P. Fedorov, B.P. Sobolev, Appl. Phys. Lett. 67, 31 (1995).

[4] A. Suchocki, B. Koziarska, T. Langer, J.M. Langer, Appl. Phys. Lett. 70, 2934 (1997).

[5] J.E. Dmochowski, J.M. Langer, Z. Kalinski, W. Jantsch, Phys. Rev. Lett. 56, 1735 (1986).

[6] W. Jantsch, Z. Wilamowski, G. Ostermayer, Semicond. Sci. Technol. 6, B47 (1991).

[7] Z. Wilamowski, H. Przybylińska, W. Jantsch, J. Magn. Magn. Mater. 140-144, 1733 (1995). 